\title{
Electronic and spin properties of hole point contacts
}

\author{
U. Zülicke*1 \\ ${ }^{1}$ Institute of Fundamental Sciences and MacDiarmid Institute for Advanced Materials and Nanotechnology, \\ Massey University, Private Bag 11 222, Palmerston North, New Zealand \\ Received PASPS-IV paper number PB-45
}

Key words hole quantum wires, anisotropic Zeeman splitting, Luttinger Hamiltonian. PACS 71.70.Ej, 73.21.Hb, 71.55.Eq

\begin{abstract}
We have studied theoretically the effect of a tuneable lateral confinement on two-dimensional hole systems realised in III-V semiconductor heterostructures. Based on the $4 \times 4$ Luttinger description of the valence band, we have calculated quasi-onedimensional (quasi-1D) hole subband energies and anisotropic Landé $g$-factors. Confinement-induced band mixing results in the possibility to manipulate electronic and spin properties of quasi-1D hole states over a much wider range than is typically possible for confined conduction-band electrons. Our results are relevant for recent experiments where source-drain-bias spectroscopy was used to measure Zeeman splitting of holes in p-type quantum point contacts.
\end{abstract}

Copyright line will be provided by the publisher

1 Introduction Low-dimensional hole systems provide an interesting playground for engineering spin properties of charge carriers. In typical semiconductors, quantum confinement affects the physical properties of holes much more strongly than those of electrons [1]. This is because subband quantisation causes an energy splitting between heavy-hole (HH) and light-hole ( $\mathrm{LH})$ bands, and modifies their residual coupling. The Zeeman splitting of 2D hole systems for in-plane magnetic fields is a good example; it is suppressed for HH states [2] but doubled for LH states [3]. On the most basic level, this behaviour can be understood by noting that the heavy and light-hole bands belong to a quadruplet of states having total angular momentum $j=3 / 2$. More detailed theory [4] based on the Luttinger description [5] of the valence band provides realistic values for the Landé $g$-factors of 2D holes, including the in-plane Zeeman-splitting anisotropy in low-symmetry heterostructures. The competition between confinement-induced band mixing and HH-LH energy splitting also determines the physical properties of hole quantum wires [6], dots [7], and localised acceptor states [8]. Recently, transport measurements in hole wires [9] and point contacts [10] have become possible, opening up new possibilities to investigate electronic and spin properties of quasi-1D hole systems. Here we investigate the confinement dependence of hole $g$-factors in quasi-1D structures, mapping the crossover between the weakly confined 2D and symmetrically confined 1D limits.

2 Theoretical description of quasi-1D hole systems The HH and LH bands are distinguished by the quantum number $j_{z}$ of projection of total angular momentum along the quantisation $(z)$ axis: $j_{z}= \pm 3 / 2$ for HHs, $j_{z}= \pm 1 / 2$ for LHs. Their dynamics is described by the Luttinger Hamiltonian [5], which forms the basis for our investigation of quasi-1D hole systems. While the effect of remote bands (splitoff, conduction, etc.) may need to be included to achieve reasonable quantitative accuracy [11], we do not expect it to change the qualitative features of the 2D-to-1D crossover focussed on in this work. In the following, we use atomic units $\left(\hbar=m_{0}=1\right)$ and adhere to the hole picture for energy bands. We also find it useful to employ a universal representation $[12,13]$ of the Luttinger Hamiltonian as a sum over tensor invariants where all information about band structure is contained in the Luttinger parameters $[5,13]$

* Corresponding author: e-mail: u.zuelicke@massey.ac.nz, Phone: +64 63505799 extn 7259, Fax: +64 63505682 
$\gamma_{1}, \gamma_{\mathrm{s}}=\left(3 \gamma_{3}+2 \gamma_{2}\right) / 5, \gamma_{\delta}=\left(\gamma_{3}-\gamma_{2}\right) / 2$, and coordinate-system-dependent constants $c_{j}$. The most general $4 \times 4$ valence-band Hamiltonian $[4,13]$ also includes terms describing spin splitting due to the Zeeman effect or arising from bulk (B) and/or structural (S) inversion asymmetry (IA). It reads

$$
\mathcal{H}_{4 \times 4}=\left[\frac{\gamma_{1}}{2}+\frac{5}{4} \gamma_{\mathrm{s}}\right] \hat{\mathbf{k}}^{2}-\gamma_{\mathrm{s}}(\hat{\mathbf{k}} \cdot \hat{\mathbf{J}})^{2}+\gamma_{\delta} \sum_{j} c_{j} \mathcal{T}_{j}(\hat{\mathbf{k}}, \hat{\mathbf{J}})+\mathcal{H}_{\mathrm{Z}}+\mathcal{H}_{\mathrm{BIA}}+\mathcal{H}_{\mathrm{SIA}}+V(\hat{\mathbf{r}}) .
$$

We denote operators of position, kinetic wave vector, and total angular momentum by $\hat{\mathbf{r}}, \hat{\mathbf{k}}$, and $\hat{\mathbf{J}}$, respectively. $\mathcal{T}_{j}(\hat{\mathbf{k}}, \hat{\mathbf{J}})$ are tensor invariants associated with axial $(j=0)$ and cubic $(j \neq 0)$ corrections to the spherical Luttinger Hamiltonian; see the notation used by Fishman [13].

In the following, we include the isotropic Zeeman term $\mathcal{H}_{\mathrm{Z}}=2 \kappa \mu_{\mathrm{B}} \mathbf{B} \cdot \hat{\mathbf{J}}$ with bulk-hole $g$-factor $\kappa$ but neglect spin splitting due to IA. We consider a rectangular hard-wall confining potential $V(\hat{\mathbf{r}})$ in the $x y$ plane, characterised by quantum-well widths $W_{x}$ and $W_{y}$. Hence we adopt the wire axis as the quantisation axis of total angular momentum. As we are interested in describing the properties of hole point contacts realised by lateral confinement in a $2 \mathrm{D} \mathrm{HH}$ system [10], we assume a strong confinement in $x$ direction and consider only the lowest orbital quantum-well bound state. Results obtained in the spherical approximation (i.e., for $\gamma_{\delta}=0$ ) serve to illustrate that the crossover between the 2D and symmetrically confined 1D limits (realised for $W_{y} \gg W_{x}$ and $W_{y} \rightarrow W_{x}$, respectively) is concomitant with a monotonous change from $\mathrm{HH}$ to LH character for the lowest quasi-1D subbands. As recent hole point contacts [10] were realised in quantum wells grown in the low-symmetry [113] direction, we also present results for quasi-1D hole states calculated from the full Luttinger Hamiltonian, including axial and cubic corrections.

3 2D-to-1D crossover in the spherical approximation In the spherical approximation for the kinetic energy, the Hamiltonian for a hole quantum wire can be written as $\mathcal{H}_{\mathrm{s}}^{(\text {wire })}=\mathcal{H}_{\mathrm{s}}^{(\mathrm{sb})}+\mathcal{H}_{\mathrm{s}}^{(\mathrm{hl})}+\mathcal{H}_{\mathrm{s}}^{(\mathrm{lD})}+\mathcal{H}_{\mathrm{s}}^{(\text {mix })}$. These terms correspond to quantised 1D bound-state energies (sb), a coupling between heavy and light holes arising from asymmetric $1 \mathrm{D}$ confinement (hl), the quadratic energy dispersion for hole motion along the wire (1D), and a term that accounts for additional inter-subband mixing between heavy and light holes (mix). Introducing total-angular-momentum ladder operators $\hat{J}_{ \pm}=\left(\hat{J}_{x} \pm i \hat{J}_{y}\right) / \sqrt{2}$, combinations $\hat{k}_{ \pm}=$ $\hat{k}_{x} \pm i \hat{k}_{y}$, and the notation $\{$,$\} for a symmetrised product of two operators, the explicit expressions are$

$$
\begin{aligned}
\mathcal{H}_{\mathrm{s}}^{(\mathrm{sb})} & =\left\langle\left(\frac{\gamma_{1}}{2}+\frac{\gamma_{\mathrm{s}}}{2}\left[\hat{J}_{z}^{2}-\frac{5}{4}\right]\right) \hat{k}_{\perp}^{2}+V(x, y)\right\rangle, \\
\mathcal{H}_{\mathrm{s}}^{(\mathrm{hl})} & =-\frac{\gamma_{\mathrm{s}}}{2}\left\langle\hat{k}_{x}^{2}-\hat{k}_{y}^{2}\right\rangle\left(\hat{J}_{+}^{2}+\hat{J}_{-}^{2}\right) \\
\mathcal{H}_{\mathrm{s}}^{(\mathrm{dD})} & =\left(\frac{\gamma_{1}}{2}-\gamma_{\mathrm{s}}\left[\hat{J}_{z}^{2}-\frac{5}{4}\right]\right) \hat{k}_{z}^{2}, \\
\mathcal{H}_{\mathrm{s}}^{(\text {mix })} & =-\gamma_{\mathrm{s}}\left(\frac{\left\langle\left\{\hat{k}_{x}, \hat{k}_{y}\right\}\right\rangle}{2 i}\left(\hat{J}_{+}^{2}-\hat{J}_{-}^{2}\right)+\sqrt{2}\left[\left\langle\left\{\hat{k}_{z}, \hat{k}_{-}\right\}\right\rangle\left\{\hat{J}_{z}, \hat{J}_{+}\right\}+\left\langle\left\{\hat{k}_{z}, \hat{k}_{+}\right\}\right\rangle\left\{\hat{J}_{z}, \hat{J}_{-}\right\}\right]\right) .
\end{aligned}
$$

It is understood that, in Eqs. (3.1), operators inside angular brackets have to be replaced by their corresponding matrix elements between the quantised levels that diagonalise $\mathcal{H}_{\mathrm{s}}^{(\mathrm{sb})}$. The term $\mathcal{H}_{\mathrm{s}}^{(\mathrm{hl})}$ mixes $\mathrm{HH}$ and LH states from the same and also between different quantised levels of $\mathcal{H}_{\mathrm{s}}^{(\mathrm{sb})}$. As we are only interested in hole-wire subbands at energies well below the 2D LH subband edge, we can neglect the HH-LH mixing between different quantised levels, in particular also those arising from $\mathcal{H}_{\mathrm{s}}^{(\mathrm{mix})}$. Quasi-1D hole-subband energies obtained within this version of the subband $\mathbf{k} \cdot \mathbf{p}$ method [14] are shown in Figure 1. We obtained analytical expressions, which we omit here because of space limitations.

In a quantum point contact [15], a conductance step occurs whenever a quasi-1D subband edge passes through the Fermi level $E_{\mathrm{F}}$. The spectroscopic determination of Zeeman splitting [16] is based on an 


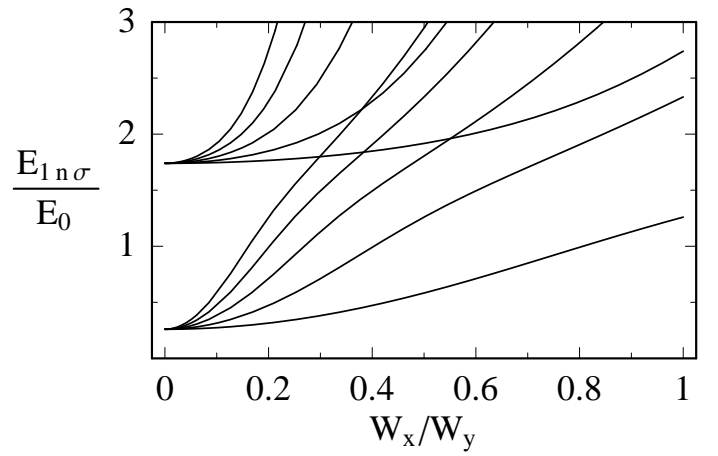

Fig. 1 Subband energies for hole wires formed by a hard-wall confinement in the $x y$ plane, characterised by widths $W_{x}$ and $W_{y}$. We show results for the five lowest wire subband edges deriving from the lowest $2 \mathrm{D} \mathrm{HH}$ and $\mathrm{LH}$ subbands, respectively. $E_{0}=$ $\gamma_{1} \pi^{2} \hbar^{2} /\left(2 m_{0} W_{x}^{2}\right)$, where $\gamma_{1}$ denotes a Luttinger parameter [5] and $m_{0}$ is the electron mass in vacuum. The bulk valence-band bottom is taken as zero energy, and $\gamma_{\mathrm{s}} / \gamma_{1}=0.37$ was assumed (the value for GaAs [17]). We neglected inter-band HH-LH coupling terms that will change subband crossings into anticrossings but do not affect wire subbands at energies well below the 2D LH level.

analysis of how these steps change with an applied magnetic field. Hence it is useful to study 1D holesubband edges. For comparison with experiments, we provide an approximate formula for $E_{\mathrm{F}}$ in a $2 \mathrm{D} \mathrm{HH}$ system with density $n_{2 \mathrm{D}}$ and quantum-well width $W_{x}$. Measured from the bulk valence-band edge, it is

$$
\frac{E_{\mathrm{F}}}{E_{0}}=1-2 \frac{\gamma_{\mathrm{s}}}{\gamma_{1}}+\frac{2}{\pi}\left(1+\frac{\gamma_{\mathrm{s}}}{\gamma_{1}}\right) n_{2 \mathrm{D}} W_{x}^{2} .
$$

Assuming $n_{2 \mathrm{D}}=1 \times 10^{15} \mathrm{~m}^{-2}$ and $W_{x}=20 \mathrm{~nm}$, which corresponds to the experimental situation of Ref. [10], we find $E_{\mathrm{F}} / E_{0}=0.61$. Using Fig. 1, we can estimate $W_{y} \approx 2 W_{x}$ at the last conductance step.

We have determined the anisotropic hole spin splitting due to an external magnetic field $\mathbf{B}$ by diagonalising the sum of the Zeeman term $\mathcal{H}_{Z}$ and the spherical Luttinger Hamiltonian with confinement [Eqs. (3.1)]. Landé $g$-factors for fields parallel to the $x, y, z$ directions can be extracted from the corresponding Zeeman spin splittings. We focus here on the states at quasi-1D subband edges, i.e., for $k_{z}=0$. It turns out that the $g$-factors are only functions of the subband energy, i.e., have no explicit dependence on the wire's aspect ratio and quasi-1D-level quantum number. Hence, within the spherical approximation, the hole $g$-factor is the same for all quasi-1D subbands when they pass the Fermi energy in a point contact. We show corresponding values in Figure 2. Again, space limitations prevent us from giving analytical results.

4 Realistic description of hole wires: Axial and cubic corrections The simple spherical model discussed in the previous Section captures the qualitative 2D-to-1D crossover expected in a hole QPC. However, for a proper quantitative description of the Zeeman splitting, we need to include axial and cubic terms as well. For example, cubic terms are providing the leading contribution to the $g$-factor for in-plane field directions in 2D HH systems [4] and, hence, can also be expected to be dominant for wide hole wires. Hence, the dependence of $g$-factors on wire width will be strongly influenced by the axial and cubic parts.

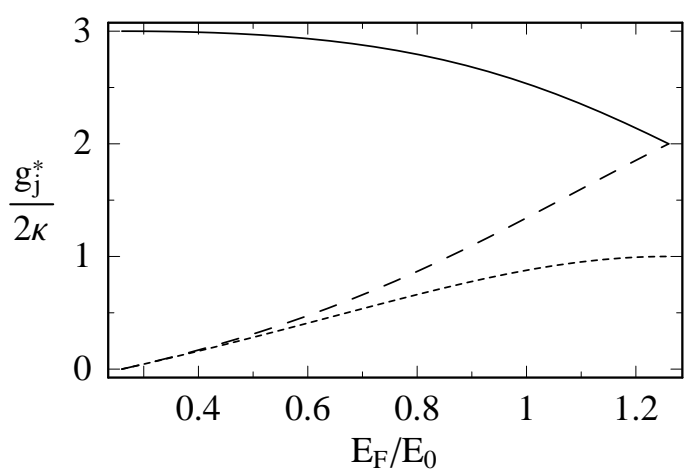

Fig. 2 Landé $g$-factors of hole-wire subband edges crossing the Fermi energy $E_{\mathrm{F}}$. The solid (dashed, dotted) curve corresponds to the situation with magnetic field applied in the $x(y, z)$ direction. (The wire is aligned with the $z$ axis and the 2D quantum well is grown in the $x$ direction.) The results shown are obtained using GaAs bandstructure parameters [17] and are valid for magnetic fields that are much smaller than the scale $\tilde{B}=\pi^{2} \hbar /\left(e W_{x}^{2}\right)$. The Fermi energy is measured from the bulk valence-band bottom. The evolution of the $g$-factor anisotropy indicates the crossover of hole-wire subbands having $\mathrm{HH}$ character at small $E_{\mathrm{F}}$ (i.e., weak lateral confinement) to $\mathrm{LH}$ character in a symmetrically confined wire. 


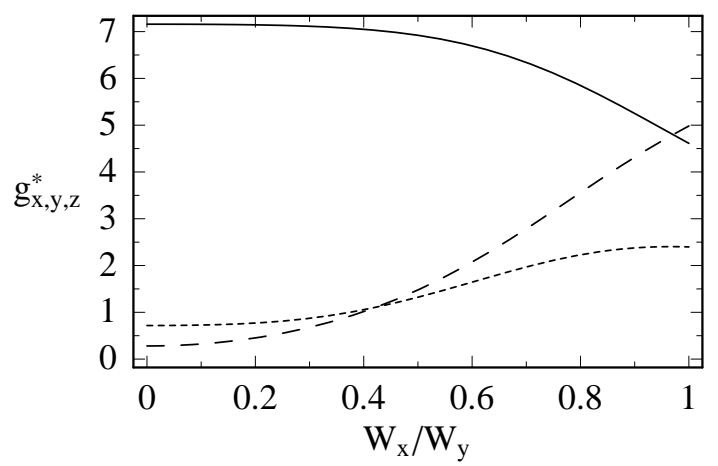

Fig. 3 Effective $g$-factors as a function of aspect ratio for a hole wire realised in a [113] GaAs quantum well and oriented parallel to the $[33 \overline{2}]$ direction. The solid (dashed, dotted) curve corresponds to the magnetic field being applied in the $x(y, z)$ direction. (We consider the geometry where the wire is aligned with the $z$ axis and the 2D quantum well is grown in the $x$ direction.) Results shown are obtained using GaAs bandstructure parameters [17] and are valid for magnetic fields that are much smaller than the scale $\tilde{B}=\pi^{2} \hbar /\left(e W_{x}^{2}\right)$ set by the 2D quantum-well confinement.

As before, we find the hole $g$-factors by diagonalising the sum of the Luttinger Hamiltonian (now including axial and cubic terms) with confinement and the Zeeman term, again neglecting HH-LH coupling between different quantised orbital levels. Our results for a quantum-wire geometry realised in recent experiment [10] are shown in Figure 3. With cubic corrections included, the $g$-factors obtained for inplane magnetic-field directions in the 2D limit $\left(W_{y} \gg W_{x}\right)$ are finite. Their values agree quite well with those given previously [4], even though our approach neglects HH-LH mixing between different quantised 1D orbital levels and, hence, could have been rather bad for describing the 2D limit. Most interestingly, though, we find that the in-plane $g$-factor anisotropy changes character during the 2D-to-1D crossover. The crossing of the dashed and dotted curves in Fig. 3 indicates that the confinement-induced HH-LH mixing eventually results in a larger Zeeman splitting for in-plane fields perpendicular to the hole wire as compared with fields parallel to the wire, effectively reversing the situation encountered in the 2D limit.

5 Conclusions We calculated quasi-1D subbands and $g$-factors for laterally confined 2D HH systems. Confinement-induced band mixing causes a strongly wire-width-dependent Zeeman splitting. Future work needs to consider the effect of a nonuniform lateral confinement and address many-body effects.

Acknowledgements The author thanks O.P. Sushkov for illuminating discussions and R. Winkler for a critical reading of this manuscript. Financial support from the Marsden Fund of the Royal Society of NZ is gratefully acknowl-

edged. This research was also supported in part by the National Science Foundation under Grant No. PHY99-07949 during a visit to the Kavli Institute for Theoretical Physics at the University of California, Santa Barbara.

\section{References}

[1] G. Bastard, J. A. Brum, and R. Ferreira, in Solid State Physics, edited by H. Ehrenreich and D. Turnbull (Academic Press, San Diego, 1991), Vol. 44, pp. 229-415.

[2] H. W. van Kesteren, E. C. Cosman, W. A. J. A. van der Poel, and C. T. Foxon, Phys. Rev. B 41, 5283 (1990).

[3] B. Kuhn-Heinrich et al., Sol. State Commun. 91, 413 (1994).

[4] R. Winkler, Spin-Orbit Coupling Effects in Two-Dimensional Electron and Hole Systems, Vol. 191 of Springer Tracts in Modern Physics (Springer, Berlin, 2003).

[5] J. M. Luttinger, Phys. Rev. 102, 1030 (1956).

[6] P. C. Sercel and K. J. Vahala, Appl. Phys. Lett. 57, 545 (1990).

[7] T. Darnhofer, D. A. Broido, and U. Rössler, Phys. Rev. B 50, 15412 (1994).

[8] K.-M. Haendel et al., Phys. Rev. Lett. 96, 086403 (2006).

[9] L. N. Pfeiffer et al., Appl. Phys. Lett. 87, 073111 (2005).

[10] R. Danneau et al., Appl. Phys. Lett. 88, 012107 (2006).

[11] D. S. Citrin and Y.-C. Chang, Phys. Rev. B 40, 5507 (1989).

[12] N. O. Lipari and A. Baldereschi, Phys. Rev. Lett. 25, 1660 (1970).

[13] G. Fishman, Phys. Rev. B 52, 11132 (1995).

[14] D. A. Broido and L. J. Sham, Phys. Rev. B 31, 888 (1985); S.-R. E Yang et al., Phys. Rev. B 32, 6630 (1985).

[15] C. W. J. Beenakker and H. van Houten, in Solid State Physics, edited by H. Ehrenreich and D. Turnbull (Academic Press, San Diego, 1991), Vol. 44, pp. 1-228.

[16] N. K. Patel et al., Phys. Rev. B 44, 10973 (1991).

[17] I. Vurgaftman, J. R. Meyer, and L. R. Ram-Mohan, J. Appl. Phys. 89, 5815 (2001). 